\title{
Paradigmas del proceso enseñanza-aprendizaje a distancia
}

\section{Paradigms of the distance learning-teaching process}

RANGEL-ROMERO, Carlos †*, ROJAS-GARNICA, Juan Carlos, FLORES-MARTÍNEZ, Guillermo y HERNÁNDEZ-LAZCANO, Ricardo

Universidad Tecnológica de Puebla

ID $1^{\mathrm{er}}$ Autor: Carlos, Rangel-Romero / ORC ID: 0000-0001-7813-5579, CVU CONACYT ID: 177117

ID $1^{\text {er }}$ Coautor: Juan Carlos, Rojas-Garnica / ORC ID: 0000-0002-2261-587X, CVU CONACYT ID: 66417

ID $2^{\text {do }}$ Coautor: Guillermo, Flores-Martínez / CVU CONACYT ID: 169853

ID $3^{\text {er }}$ Coautor: Ricardo, Hernández-Lazcano / ORC ID: 0000-0003-1142-2930, CVU CONACYT ID: 920171

DOI: $10.35429 /$ JTAE.2020.11.4.22.25

Recibido: 20 de Marzo, 2020; Aceptado 27 de Junio, 2020

\section{Resumen}

La enseñanza y el aprendizaje en línea constituye un paradigma alternativo a la educación presencial. Promueve la generación del conocimiento a través de procesos teóricos y metodológicos derivados del desarrollo de la ciencia y la tecnología en el campo de la comunicación y la información. Con la educación a distancia, el docente emprende estrategias innovadoras que promuevan el aprendizaje significativo. En este paradigma, el docente como sujeto educativo no se excluye, por el contrario, asume un nuevo rol que transita hacia la nueva cultura de la educación a distancia con base a la diversidad de recursos que tiene la Web. Los desafíos más relevantes de este nuevo paradigma son: la accesibilidad, el sistema personalizado, la flexibilidad en el estudio, y la interactividad con mejores materiales y recursos de aprendizaje.

\section{Enseñanza, Aprendizaje en línea, Sistema personalizado}

\begin{abstract}
Online teaching and learning are an alternative paradigm to face-to-face education. It promotes the generation of knowledge through theoretical and methodological processes derived from the development of science and technology in the field of communication and information. With distance education, the teacher undertakes innovative strategies that promote meaningful learning. In this paradigm, the teacher as an educational subject is not excluded, on the contrary, it assumes a new role that transits towards the new culture of distance education based on the diversity of resources that the Web has. The most relevant challenges of this new paradigm are accessibility, personalized system, flexibility in study, and interactivity with better learning materials and resources.
\end{abstract}

Teaching, Online Learning, Personalized System

Citación: RANGEL-ROMERO, Carlos, ROJAS-GARNICA, Juan Carlos, FLORES-MARTíNEZ, Guillermo y HERNÁNDEZ-LAZCANO, Ricardo. Paradigmas del proceso enseñanza-aprendizaje a distancia. Revista de Tecnología y Educación. 2020. 4-11: 22-25

\footnotetext{
* Correspondencia del Autor (Correo electrónico: carlos.rangel@utpuebla.edu.mx)

$\dagger$ Investigador contribuyendo como primer autor.
} 


\section{Introducción}

Un paradigma en la educación es todo aquello que posee un método el cual ya está definido y está compuesto de supuestos teóricos y formas de aplicación a la realidad con el único fin de transformarlas. Es por ello que un paradigma en la educación en línea propone una manera diferente de concebir el proceso de enseñanza aprendizaje, así como también, el discurso educativo y todas sus dimensiones que involucran a la enseñanza. Es por ello que, el proceso de enseñanza-aprendizaje en línea debe contemplar como objetivo principal formar personas que sean capaces de aprender a aprender en forma permanente y así los alumnos obtendrán habilidades y competencias para entender los diferentes problemas que se encontrarán en su vida profesional y resolverlos de acuerdo a su conocimiento adquirido. El proceso de enseñanza-aprendizaje en línea se puede comprender como una disciplina en donde conviven varios paradigmas alternativos con los cuales el docente se encuentra en disputa para poder desarrollar el método más adecuado para el desarrollo del proceso de enseñanza.

\section{Los nuevos retos del docente}

Actualmente el docente también tiene que hacer suyos los paradigmas de aprender a estudiar, por lo cual, tiene que poner en práctica a aprender a desaprender, porque tiene que adecuarse a los tiempos actuales sobre la generación de conocimiento, y tiene que aprender a emprender para generar nuevas estrategias y métodos de investigación que generen conocimientos y con ello aprenden a arriesgarse con lo cual generará su propio conocimiento y desarrollará nuevas estrategias para el proceso de enseñanzaaprendizaje. Todo ello, con la única finalidad de poder hacer frente a la nueva situación en la sociedad del conocimiento.

Hoy en día, con la educación a distancia el docente tiene el gran reto de consolidar los procesos de enseñanza para que el alumno obtenga un conocimiento significativo, ya que es importante su rol como impulsor para generar un conocimiento a partir del pensamiento teórico y conceptual. Pero para tener éxito en el proceso de enseñanza a distancia requiere de una innovación, destrezas y estrategias que permitan obtener un panorama real sobre el proceso de aprendizaje de los alumnos, y así aportar determinadas soluciones basadas en el aprendizaje.
En esta coyuntura, los paradigmas del proceso de enseñanza a distancia atiende a las nuevas formas educativas con un cambio de acento en cuanto al sujeto de la acción, que incide directamente en la importancia y necesidad que tienen los estudiantes de obtener un conocimiento y hace a un lado la forma de enseñar que tenía en forma exclusiva por parte del docente, ya que hoy en día su función es ser un guía y de impulsor de aprendizajes, con esta función la figura del docente no se excluye pero si requiere de integrarse y sobre todo reflexionar lo que adquiere su importancia en su nuevo rol, que es el observar al estudiante y desarrollar su conocimiento de acuerdo con sus capacidades que, además, consiga que se transformen en un valor añadido tangible, práctico y activo.

Con base a ello, actualmente se cuenta con esos medios que facilitan la educación a distancia como es la WEB que permite la creación de conocimiento en grupo, el cual es de forma colaborativa, mediante recursos compartidos como son blogs, YouTube, Facebook, correo electrónico personal, entre otras más, los cuales favorecen el desarrollo de competencias relacionadas con la capacidad de comunicarse, que son hablar, escribir, leer, comprender, interpretar y como consecuencia desarrollar el pensamiento crítico. Con ello se logra desarrollar la autonomía, la iniciativa, la capacidad para el trabajo en grupo, asi como la responsabilidad individual, todas esas capacidades son competencias que el mismo alumno genera para obtener un conocimiento.

\section{Se puede aprender a distancia}

Hoy en dia la educación a distancia es más factible ya que ha venido de la mano por el avance tecnológico en estos últimos años, es por ello que es importante incorporar a las tecnologías de la información como elemento mediador entre el docente y el alumno, ya que esto permite una percepción más clara acerca de la educación a distancia como una alternativa real a la educación presencial.

El principal objetivo de la educación a distancia es hacer llegar el conocimiento a todo aquel que lo necesita. Sin embargo, en años pasados la educación a distancia era considerada como una educación de carácter compensatorio, a lo cual estaban condenados todos los estudiantes que no podían asistir a la educación presencial. 
Pero el avance tecnológico en las tecnologías de la información y la comunicación, han hecho que la educación a distancia se considere como una alternativa real a la educación presencial. Es por ello que el empleo intensivo de las tecnologías de la información ha permitido una percepción más clara y más entendible de este tipo de educación. Es importante mencionar que desde una concepción basada en la idea fundamental de que para lograr un aprendizaje era necesario únicamente el contacto entre el docente y el estudiante como una actividad necesaria para lograr una educación, de base oral, con un uso escaso de la lengua escrita y los libros para desarrollar un aprendizaje, constituyendo la base de la educación presencial. Pero en la educación a distancia las redes tecnológicas permiten la interacción no solo entre los estudiantes, sino también entre docentes y fuentes de información para acumular conocimiento de manera progresiva y asi desarrollar habilidades cognitivas.

Las ventajas de los trabajos en red hacen mucho énfasis en las oportunidades y recursos disponibles para los estudiantes y docentes. Ya que con el empleo de las redes tecnológicas se logra tener acceso a libros, bibliotecas, y bases de datos en el mundo. La diferencia mas importante entre la educación presencial y la educación a distancia reside en el cambio de medio y en el potencial educativo que se deriva de la optimización del uso de cada medio.

En este sentido, cabe realizar una reflexión sobre el hecho de que los modelos virtuales en la educación a distancia no tendrá éxito si se basan en replicar los modelos presenciales, es por ello que será necesario una adaptación que aproveche lo mejor que ese medio ofrece y que de esta forma alcance los mismos objetivos formativos que se plantean en una clase presencial, y es ahí donde la educación a distancia y sus diferentes aportes didácticos pueden realizar un aporte diferente al de una clase presencial.

Es importante recalcar que el reto de la educación a distancia debe ser el mismo reto de la educación presencial, que es el incrementar el nivel de calidad de formación de estudiantes y a la vez promover la investigación necesaria para desarrollar un conocimiento propio.

\section{Cuáles serían los retos de educación a distancia}

El primero sería el promover la accesibilidad, que todos los estudiantes tengan acceso al internet, a una computadora y ofrecerlas en conjunto a la comunidad para su máximo aprovechamiento, ya que la tecnología está proveyendo cada día de nuevas posibilidades de acceder a un conocimiento a través de los sistemas de comunicación.

También es necesario contribuir a la obtención de un sistema educativo a distancia mucho más personalizado, ya que se trata de facilitar de una manera individual a las necesidades formativas de cada estudiante y así diseñar una estrategia según el estilo de aprendizaje de cada alumno.

Desarrollar en el alumno una flexibilidad en sus hábitos de estudio, y es la respuesta a la adaptación de las necesidades diversas de un estudiante, es decir, tiene que adaptarse a lo que más le motiva, que se adapte a sus ritmos y expectativas, todo ello está pensado para satisfacer a las necesidades de aprendizaje de los alumnos.

El docente de educación a distancia tiene el gran reto de desarrollar sistemas tecnológicos que permitan elaborar materiales y recursos con altos niveles de interactividad entre los estudiantes, recursos con los cuales realmente sea posible convertir en información interesante, aunque sea mera información en un verdadero conocimiento de los alumnos.

Los estudiantes no están solos en su proceso de aprendizaje, cooperar con otros compañeros realizando trabajo en equipo, con los docentes creando grupos de interés con personas diversas del mismo salón de clases, pero siempre será importante traspasar las fronteras del aula, y de la universidad, todo esto deber ser acompañado por el docente mediante un proceso personalizado.

Tambien será importante realizar un esfuerzo para alcanzar estándares de calidad que permitan establecer criterios para la acreditación de la educación desarrollada en contextos no presenciales de aprendizaje. 


\section{Conclusiones}

Con los avances actuales en el campo de las tecnologías de la informática, la comunicación y el desarrolo del internet, la educación a distancia es una alternativa real a la educación presencial. Genera nuevos retos en los roles de docentes y estudiantes para aprender a estudiar a través de diseño de estrategias innovadoreas e interactivas. En el caso del alumno sus retos principales son el ser autodidáctica, tener la disciplina de hacer investigación en los temas de interés y tener mayor participación en las clases virtuales. En el caso del docente sus retos son tener la iniciativa por actualizarse en las nuevas herramientas educativas y enfatizar el dominio de los temas indagando en las publicaciones recientes.

La educación a distancia deja de ser un programa compenstorio y se convierte en una verdadera opción y sistema de redes que crean comunidades de aprendizaje en lo colectivo y en lo individual. Los retos que hoy presenta la educación a distancia son la de ampliar la accesibilidad, sistemas personalizados de formación, flexibilidad de hábitos de estudio y el desarrollo de la interactividad entre docentes y estudiantes.

\section{Referencias}

Alarcón, R. (2014). Conferencia Inaugural Universidad 2014, 9no Congreso Internacional de Educación Superior. La Habana: MES.

Azzurro, A., Girón, P. y Cura, R.O. (2014). "Articulación entre docencia e investigación de la enseñanza en entorno industrial". En VIII Congreso Iberoamericano de Docencia Universitaria y Educación Superior.

García J. (2011). Modelo educativo basado en competencias: Importancia y necesidad. Vol.11, No.3. pp.1-24.

Del Rey, A.; Sánchez, J. (2011). Crítica de la educación por competencias. Universitas. (15). Pp. 233-246. Revista de Ciencias Sociales y Humanas.

Estrada, John. (2012). La formación por competencias y el mundo del trabajo: de la calificación a la empleabilidad. Revista Salud pública, (1). Pp.98-111.
Fernández, Eduardo. (2009). El discurso de la formación basada en competencias profesionales. Un análisis crítico de la formación inicial de 'profesionales en la Educación Superior. REIFOP, (1), PP.: 151-160.

Filmus, D. (1994). El papel de la Educación frente a los desafíos de las transformaciones científico-tecnológicas. Publicado con autorización de los editores. Presentado Volumen II, Módulo V del Curso de Formación de Administradores de la Educación, publicado en Buenos Aires por el Ministerio de Cultura y Educación de la República Argentina, la Facultad Latinoamericana de Ciencias Sociales (FLACSO-Buenos Aires) y la Organización de Estados Iberoamericanos para la Educación, la Ciencia y la Cultura (OEI), del libro "Para qué sirve la escuela". Tesis. Grupo editorial Norma. Buenos Aires. Recuperado el 28 de febrero de 2011

Ibáñez, C. (2007). Diseño curricular basado en competencias profesionales: una propuesta desde la psicología interconductual. Revista de Educación y Desarrollo, Número XIX (6). Recuperado el 27 de febrero de 2011. 